\title{
Factors Affecting the Improvement of the Initial Peak Urinary Flow Rate after Transurethral Resection of the Prostate or Photoselective Vaporization of the Prostate for Treating Benign Prostatic Hyperplasia
}

\author{
Hwa Sub Choi, Dong Jun Kim, Dong Suk Kim, Kyoung Pil Jeon, Tae Yoong Jeong \\ Department of Urology, Kwandong University College of Medicine, Goyang, Korea
}

\begin{abstract}
Purpose: We evaluated the factors that affect the improvement of the initial peak flow rate after transurethral resection of the prostate (TURP) or photoselective vaporization of the prostate (PVP) for benign prostatic hyperplasia (BPH) patients by using noninvasive tools.

Methods: One hundred and twenty seven BPH patients who had undergone TURP or PVP between January 2005 and May 2009 were evaluated. They were divided into 2 groups: the postoperative initial peak urinary flow rate (Qmax) was less than 10 $\mathrm{mL} / \mathrm{sec}$ (Group 1; $\mathrm{n}=37$, TURP = 11, PVP=26) and more than $10 \mathrm{~mL} / \mathrm{sec}$ (Group 2; $\mathrm{n}=90$, TURP =41, PVP=49). We confirmed the patients' preoperative check lists. The check list were the international prostate symptom score (IPSS), the quality of life score, a past history of acute urinary retention (AUR), body mass index and/or pyuria, the serum prostate-specific antigen (PSA) level and the prostate volume, the prostate transitional zone volume and prostatic calcification. The initial Qmax was measured at the outpatient clinic one week after discharge.

Results: The improvement rate was not significant difference between the TURP group (78.8\%) and the PVP group (65.3\%). The efficacy parameters were the IPSS-storage symptom score, the prostate volume, the PSA level and a past history of AUR. The IPSS-storage symptom scores of Group $1(12.3 \pm 3.3)$ was higher than those of Group $2(10.5 \pm 1.7)$. The prostate volume of Group $2(42.3 \pm 16.6 \mathrm{~g})$ was bigger than that of Group $1(36.6 \pm 7.8 \mathrm{~g})$. The PSA level of Group $2(3.8 \pm 2.6 \mathrm{ng} / \mathrm{mL})$ was higher than that of Group $1(2.6 \pm 2.6 \mathrm{ng} / \mathrm{mL})$. A past history of AUR in Group 1 (35.1\%) was more prevalent than that of Group 2 (15.6\%).

Conclusions: The non-invasive factors affecting the initial Qmax after TURP or PVP were the IPSS-storage symptom score, the prostate volume and a past history of AUR. Accordingly, in patients who have a higher IPSS-storage symptom score, a smaller prostate volume and a history of AUR, there might be a detrimental effect on the initial Qmax after TURP or PVP. These factors might also be used as long-term prognostic factors.
\end{abstract}

Keywords: Transurethral resection of the prostate; Laser therapy; Prostatic hyperplasia

\section{INTRODUCTION}

Benign prostatic hyperplasia (BPH) commonly occurs in elderly men, and it is a disease whose prevalence increases with age. The treatments for $\mathrm{BPH}$ are divided into drug treatment and surgical treatment. For the surgical treatment, transurethral resection of the prostate (TURP) has been considered as a standard treatment up to the present [1]. Photoselective vaporization of the prostate (PVP) was introduced in the late 1990s and many studies have compared it with TURP. As a result, it has
Corresponding author: Tae Yoong Jeong

Department of Urology, Myongji Hospital, Kwandong University College of

Medicine, 697-24 Hwajeong-dong, Deogyang-gu, Goyang 412-826, Korea

Tel: +82-31-810-6150 / Fax: +82-31-810-5109

E-mail : urojeong@kwandong.ac.kr

Submitted: March 9, 2011 / Accepted after revision: March 20, 2011
This is an Open Access article distributed under the terms of the Creative Commons Attribution Non-Commercial License (http://creativecommons.org/licenses/by-nc/3.0/) which permits unrestricted non-commercial use, distribution, and reproduction in any medium, provided the original work is properly cited. 
been confirmed that these two treatments showed similar effects and complications $[2,3]$.

For the case of TURP, the success rate has been reported to be $70-90 \%$ [4]. Yet in $10-30 \%$ of patients, the symptoms are not improved following the surgery. Accordingly, several studies have been conducted to predict the surgical treatment outcomes. As the most objective and reliable test, studies have shown that it is important to clarify the degree of the outlet obstruction of the bladder by conducting a pressure flow study [5]. In addition, a recent study has shown the presence of an overactive bladder as well as outlet obstruction of the bladder in many patients through a urodynamic study (UDS) [6]. However, a pressure flow study and a UDS study are very invasive tests, and so there are limitations that both tests cannot be performed for all the patients.

The peak urinary flow rate (Qmax) is the objective indicator based on which the postoperative treatment outcomes can be evaluated. According to a Korean study where long-term follow up was performed for the Qmax, it reached the maximal level at the 3 rd or 6 th postoperative month. As the time elapsed, the Qmax showed a slightly decreasing tendency [7]. According to another study in which follow-up studies were performed during a 7-year postoperative follow-up period, the Qmax was not notably continuously decreased after a 1-year postoperative period [8]. Based on these findings, it is accepted that the Qmax has long-term prognostic value for patients who undergo TURP or PVP.

We conducted this study to identify the factors affecting the Qmax in the initial stage after TURP or PVP, based on non-invasive preoperative markers.

\section{MATERIALS AND METHODS}

A total of 275 patients underwent TURP or PVP at our medical institution due to BPH during a period ranging from January of 2005 to May of 2009. The exclusion criteria included patients who did not have all their preoperative parameters assessed, those who had the concurrent presence of causative diseases such as urinary bladder stone, urethral stone and urethral stenosis that caused urinary tract obstruction, those who had prostate cancer that was confirmed postoperatively, those who had neurogenic bladder and had a past history of prostate surgery. As a result, a total of 127 patients $(\mathrm{TURP}=52, \mathrm{PVP}=72)$ were enrolled in the current study.

The patients were divided into two groups based on the post- operative Qmax; we used a cut-off value of $10 \mathrm{~mL} / \mathrm{sec}$, which implies the presence of outlet obstruction of the urinary bladder. The group with a $\mathrm{Qmax}<10 \mathrm{~mL} / \mathrm{sec}$ represented a lack of the improvement (Group 1; $\mathrm{n}=37, \mathrm{TURP}=11, \mathrm{PVP}=26$ ) and the groups with a Qmax $>10 \mathrm{~mL} / \mathrm{sec}$ represented some improvement (Group 2; $\mathrm{n}=90$, TURP $=41, \mathrm{PVP}=49$ ). A retrospective study was then conducted.

The preoperative parameters included a prostate-specific antigen (PSA) test, the international prostate symptom score (IPSS), a quality of life score (QoL), the Qmax based on uroflowmetry, the prostate volume, the transitional zone volume (TZV) and the presence of prostatic calcification under the guidance of transrectal ultrasonography (TRUS) and the body mass index (BMI) based on the height and weight. Of these, the IPSS was compared based on storage symptoms (questions 2, 4 and 7), voiding symptoms (questions 1, 3, 5, and 6) and the total scores. The TZV was assessed in a total of 127 patients, and the TZV measured in only 53 of these patients (TURP $=11$, $\mathrm{PVP}=42$ ). The presence of calcification was determined in the cases in which there was a marked presence of calcified findings with strong echogenicity and a diameter of $>3 \mathrm{~mm}$ and aposterior acoustic shadow regardless of the location of the calcification. The presence of pyuria and a past history of acute urinary retention (AUR) were also confirmed. Pyuria was defined as cases in which more than five white blood cells were observed on high-magnification light microscopy. All surgeries were performed by three surgeons who overcame the learning curve. As for the surgical methods, the surgery was done using a 24Fr. Wolf resectoscope in the TURP group based on the methods that were generally described in a textbook [9]. In the PVP group, a potassium-titanyl-phosphate laser with a mean output of 80 was used and the surgical procedure was performed at a gap of 0.5-1 mm between the laser and the tissue (the near contact technique). We performed TRUS guided prostate needle biopsies in all the patients with an elevated PSA level $(\geq 4$ $\mathrm{ng} / \mathrm{mL}$ ). All the patients postoperatively had a $22 \mathrm{Fr}$. Foley catheter inserted. The catheter was removed on the day of discharge. Then, the patients were confirmed to be able to perform selfvoiding. The initial Qmax was measured at the outpatient clinic one week after discharge.

In the current study, the parameters that showed significance for the total population were also separately analyzed in the TURP and PVP group.

Statistical analysis was performed with Student's t-test and the chi-square test. A P-value $<0.05$ was considered statistically 


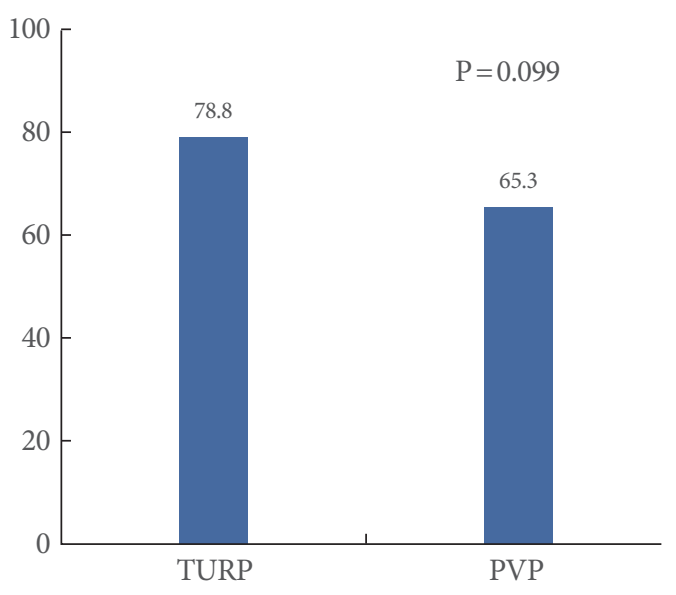

Fig. 1. Improvement rate of postoperative peak urinary flow rate in both group. Qmax, peak urinary flow rate; TURP, transurethral resection of the prostate; PVP, photoselective vaporization of the prostate.

significant. Statistical analysis was performed using SPSS ver. 12.0 (SPSS Inc., Chicago, IL, USA).

\section{RESULTS}

Following the two types of surgery (TURP and PVP), the number of patients who improved an initial Qmax to $>10 \mathrm{~mL} / \mathrm{sec}$ postoperatively was 41 in the TURP group (78.8\%) and 49 in the PVP group (65.3\%). These results indicate that the rate of improvement was slightly higher in the TURP group as compared with that of the PVP group, but the difference was not statistically significant $(\mathrm{P}>0.05)$ (Fig. 1).

The age was higher in Group $1(71.3 \pm 7.1$ years) than that in Group 2 (69.3 \pm 7.4 years). Preoperatively, the Qmax was higher in the Group $2(8.4 \pm 4.5 \mathrm{~mL} / \mathrm{sec})$ than that in the Group $1(7.3$ $\pm 5.9 \mathrm{~mL} / \mathrm{sec}$ ), but the differences were not statistically significant. The BMI and the presence of pyuria were found to have a similar profile in both groups and the differences were not statistically significant.

The IPSS-total score, the voiding symptom score and the QoL questionnaire showed no significant difference between the two groups. But the storage symptom score was found to be $12.3 \pm$ 3.3 points in Group 1 and $10.5 \pm 1.7$ points in Group 2. The storage symptom score was significantly lower in Group 2 (Table 1).

In regard to the prostatic factors based on TRUS, there were no significant differences of the transitional zone index and prostatic calcification between the two groups. However, the
Table 1. Perioperative characteristics and perioperative date

\begin{tabular}{lccc}
\hline & \multicolumn{2}{c}{ Patient } & P-value \\
\cline { 2 - 3 } & Group 1 (n=37) & Group 2 (n=90) & \\
\hline Age (yr) & $71.3 \pm 7.1$ & $69.3 \pm 7.4$ & 0.160 \\
Qmax $(\mathrm{mL} / \mathrm{sec})^{\mathrm{a})}$ & $7.3 \pm 5.9$ & $8.4 \pm 4.5$ & 0.257 \\
IPSS-storage & $12.3 \pm 3.3$ & $10.5 \pm 1.7$ & $0.003^{\mathrm{b}}{ }^{\mathrm{y}}$ \\
IPSS-voiding & $12.0 \pm 5.2$ & $13.4 \pm 4.3$ & 0.128 \\
IPSS-total & $24.4 \pm 6.3$ & $23.9 \pm 5.1$ & 0.677 \\
QoL & $4.3 \pm 0.8$ & $4.3 \pm 1.0$ & 0.983 \\
Prostate volume (g) & $36.6 \pm 7.8$ & $42.3 \pm 16.6$ & $\left.0.009^{\mathrm{b}}\right)$ \\
TZV (g) (n=53) & $16.9 \pm 9.8$ & $23.9 \pm 13.6$ & 0.138 \\
TZI (\%) (n=53) & $44.6 \pm 18.2$ & $49.9 \pm 10.0$ & 0.315 \\
PSA (ng/mL) & $2.6 \pm 2.6$ & $3.8 \pm 2.6$ & $0.037^{\mathrm{b})}$ \\
BMI & $24.3 \pm 3.3$ & $24.5 \pm 3.0$ & 0.716 \\
AUR & 35.1 & 15.6 & $0.014^{\text {b) }}$ \\
Prostatic calcification & 32.4 & 26.7 & 0.512 \\
Pyuria & 16.2 & 15.6 & 0.926 \\
\hline
\end{tabular}

Values are presented as mean $\pm \mathrm{SD}$ or $\%$.

Group 1, initial Qmax < $10 \mathrm{~mL} / \mathrm{sec}$; Group 2, initial Qmax $\geq 10 \mathrm{~mL} /$ sec; Qmax, peak urinary flow rate; IPSS, international prostate symptom score; QoL, quality of life; TZV, transitional zone volume; TZI, transitional zone index; PSA, prostate specific antigen; BMI, body mass index; AUR, acute urinary retention.

${ }^{\text {a) }}$ Preoperative Qmax. ${ }^{\mathrm{b})} \mathrm{P}<0.05$.

prostate volume was $36.6 \pm 7.8 \mathrm{~g}$ in Group 1 and $42.3 \pm 16.6 \mathrm{~g}$ in Group 2. The prostate volume was significantly higher in Group 2 (Table 1).

The PSA level was $2.6 \pm 2.6 \mathrm{ng} / \mathrm{mL}$ in Group 1 and $3.8 \pm 2.6$ $\mathrm{ng} / \mathrm{mL}$ in Group 2. The proportion of patients who had a past history of acute urinary retention was found to be $35.1 \%$ in Group 1 and $15.6 \%$ in Group 2. These results indicate that the PSA level was higher and the proportion of patients who had a past history of acute urinary retention was lower in Group 2, and these differences were statistically significant (Table 1).

We performed inter-group comparison for the IPSS-storage score, the prostate volume, the PSA level and a past history of AUR, which were all found to be statistically significance in all the patient groups and it showed that the prostate volume (37.2 \pm 7.7 g vs. $48.3 \pm 20.0 \mathrm{~g}$ ) showed a significant difference in the TURP group (Table 2). In the PVP group, the IPSS storage score $(12.5 \pm 3.1$ points vs. $10.8 \pm 1.8$ points) and the PSA level $(1.9 \pm$ $2.1 \mathrm{ng} / \mathrm{mL}$ vs. $3.4 \pm 2.3 \mathrm{ng} / \mathrm{mL}$ ) were found to be significantly different. (Table 3). 
Table 2. Comparisons of factors that showed significance in the total population at TURP group

\begin{tabular}{lccc}
\hline & \multicolumn{2}{c}{ Patient } & P-value \\
\cline { 2 - 3 } & Group 1 (n=11) & Group 2 $(\mathrm{n}=41)$ & \\
\hline IPSS-storage & $11.9 \pm 3.8$ & $10.0 \pm 1.4$ & 0.151 \\
Prostate volume (g) & $37.2 \pm 7.7$ & $48.3 \pm 20.0$ & $0.007^{\text {a) }}$ \\
PSA (ng/mL) & $4.6 \pm 3.0$ & $4.2 \pm 2.8$ & 0.722 \\
AUR & 36.4 & 14.6 & 0.104 \\
\hline
\end{tabular}

Values are presented as mean \pm SD or $\%$.

TURP, transurethral resection of the prostate; Group 1, initial Qmax $<10 \mathrm{~mL} / \mathrm{sec}$; Group 2, initial Qmax $\geq 10 \mathrm{~mL} / \mathrm{sec}$; IPSS, international prostate symptom score; PSA, prostate specific antigen; AUR, acute urinary retention.

${ }^{\text {a) }} \mathrm{P}<0.05$.

\section{DISCUSSION}

To date, several studies have been conducted to predict the treatment outcomes of TURP, which is routinely performed in patients with BPH. Yet in regard to PVP, it is difficult to identify the treatment outcomes. In the current study, a retrospective analysis was performed by considering the two treatment modalities as a single modality based on several recent reports showing that the treatment outcomes are similar between PVP and TURP $[2,3]$.

In the recent studies that focused on the prognostic factors of the treatment outcomes of TURP, it was important to evaluate the degree of the outlet obstruction of the bladder by using UDS or a pressure flow study. These studies were conducted using objective data. Yet it is true that a UDS or a pressure flow study cannot be performed for all the patients because of the studies' invasiveness. Accordingly, in the current study, a UDS and a pressure flow study were not performed. Instead, based on the preoperative characteristics of the patients that were found using such non-invasive methods as TRUS, a lab study and physical examination, we attempted to examine the factors affecting the Qmax in the early phase of the postoperative stage.

In regard to the prostate volume, many controversial opinions exist regarding its correlation with the voiding symptoms of patients. Jensen et al. [10] reported that the degree of the improvement of symptoms is not affected by the prostate volume following surgery. According to Cockett et al. [11] on the preoperative work-up of patients with $\mathrm{BPH}$, as the prostate volume was greater, the postoperative outcomes were better. Also in our study, the prostate volume was $42.3 \pm 16.6 \mathrm{~g}$ in Group 2 and $36.6 \pm 7.8 \mathrm{~g}$ in Group 1. There was a discrepancy by approxi-
Table 3. Comparisons of factors that showed significance in the total population at PVP group

\begin{tabular}{lccl}
\hline & \multicolumn{2}{c}{ Patient } & P-value \\
\cline { 2 - 3 } & Group 1 $(\mathrm{n}=26)$ & Group 2 $(\mathrm{n}=49)$ & \\
\hline IPSS-storage & $12.5 \pm 3.1$ & $10.8 \pm 1.8$ & $0.019^{\mathrm{a})}$ \\
Prostate volume (g) & $36.3 \pm 7.9$ & $37.2 \pm 10.6$ & 0.675 \\
PSA & $1.9 \pm 2.1$ & $3.4 \pm 2.3$ & $0.007^{\mathrm{b})}$ \\
AUR & 34.6 & 16.3 & 0.072 \\
\hline
\end{tabular}

Values are presented as mean \pm SD or $\%$.

PVP, photoselective vaporization of the prostate; Group 1, initial Qmax $<10 \mathrm{~mL} / \mathrm{sec}$; Group 2, initial Qmax $\geq 10 \mathrm{~mL} / \mathrm{sec}$; IPSS, international prostate symptom score; PSA, prostate specific antigen; AUR, acute urinary retention.

${ }^{\text {a) }} \mathrm{P}<0.05 .{ }^{\text {b) }} \mathrm{P}<0.05$.

mately $5.7 \mathrm{~g}$ on average. In association with this, as the prostate volume was relatively greater, there was a higher possibility for the occurrence of outlet obstruction of the bladder. This might be similar to the reports by Jung et al. [6] that the treatment outcomes were more satisfactory following TURP in the group where there was outlet obstruction of the bladder as compared with the treatment outcomes of the group without outlet obstruction of the bladder.

IPSS is a fundamental tool by which the lower urinary tract symptoms can be quantitatively measured. Hakenberg et al. [12] examined the changes in the IPSS prior to and following TURP based on a classification into a storage symptom score and a voiding symptom score. As a result, the storage symptom score was decreased by 3.4 points and the voiding symptom score was decreased by 6.2 points. Based on these results, the decremental degree of the voiding symptom score was relatively lower than that of the storage symptom score.

Im et al. [13] reported that the satisfactory of TURP outcome decreased when storage symptom score was superior to voiding symptom score. Also in our study, it was confirmed that the mean preoperative storage symptom score was higher by 1.8 points in Group 1, in which the symptoms were not improved. These results indicate that the IPSS storage symptom score is an indicator that should be evaluated prior to TURP or PVP.

AUR is one of the serious complications that occur following the onset of BPH. It may occur due to such factors as excessive water drinking, alcohol intake, an anti-histamine intake and the aggravation of the systemic status. According to Chang et al. [14] the age, the prostate volume, the transition zone volume and its index (TZI) can be used as prognostic indicators. Yet to date, no studies have examined whether a past history of AUR 
can affect the improvement of symptoms that occur following TURP or PVP. In the current study, the proportion of patients with a past history of AUR was $15.6 \%$ in Group 2. This was lower than $35.1 \%$ in Group 1, in which the symptoms were not improved following TURP or PVP. Jang et al. [15] reported that the detrusor muscle was overstretched and its contractility was reduced in patients with chronic urinary retention. Those authors noted that the voiding functions might be difficult in these patients despite prostatectomy. The accurate reasons for this cannot be clarified. It is assumed that there is a higher possibility for the progression to chronic urinary retention in patients with a past history of AUR because there is a higher degree of sensitivity to the factors that aggravate the AUR. Thus, it is assumed that an assessment of the status of the urinary bladder would be essential with the use of a UDS and cystoscopy prior to performing TURP or PVP in patients with BPH and who have a past history of AUR.

In the current study, the PSA level was interpreted to be a significant factor affecting the improvement of symptoms. According to a Korean study where the correlation between the prostate volume and PSA was assessed, Cho et al. [16] reported that the prostate volume was correlated with the PSA level in a logarithmical manner. Those authors also noted that the rate of an increased prostate volume was relatively higher as time elapsed. In the current study, a comparison was made for age and the PSA level between the two groups. Then the results were also compared with those reported by Cho et al. [16]. This showed the similar results, based on which it is assumed that the PSA level is not a factor affecting the initial Qmax, but it should be considered to be the difference in PSA level due to that in the prostate volume between the two groups. According to a study that examined the factors affecting the improvement of symptoms following TURP, Kim et al. [17] reported that the improvement of symptoms was decreased in cases in which calcification was present in the central region of the prostate gland. According to Lee et al. [18] satisfactory surgical outcomes could be expected in cases in which the TZI was relatively higher as compared with those case with a lower TZI. In the current study, both calcification of the prostate and the TZI seemed to be statistically insignificant for the initial Qmax after TURP or PVP. Based on these findings, the presence of calcification was solely confirmed without confirming the location of calcification in the cases with calcification of the prostate. However, for the cases with TZI, the number of patients for which the TZI was measured at our medical institution was relatively smaller than the previous studies, and this might be one of the limitations of the current study.

In the current study, the factors affecting the Qmax in the initial stage following TURP or PVP were found to include the IPSS-storage symptom score, the prostate volume, the PSA level and a past history of AUR. When these factors were confirmed in both the TURP group and the PVP group, it was found that the prostate volume was a significant factor in the TURP group, and the IPSS-storage symptom score and PSA level were significant factors in the PVP group. However, these results might have come about from the fact that TURP was frequently performed in the patients with a greater volume of the prostate as compared to the PVP group and the number of total number of subject patients was relatively small. So, this finding cannot be generalized, which is also one of the limitations of the current study. Further large-scale studies are warranted to examine the factors affecting TURP and PVP, and studies with long-term follow-up are also needed.

The current study is not devoid of other limitations. TURP and PVP showed similar result, but they are different procedures. So, the idea that they have same preoperative factors affecting treatment result has a burden. Procedures were performed three surgeons and this study didn't consider the resected volume of prostate in TURP. However, despite these biases, we think that this study can be helpful in predicting the result of $\mathrm{BPH}$ surgery preoperatively.

In conclusion, following TURP or PVP, which are both commonly performed to surgically treat patients with $\mathrm{BPH}$, the noninvasive factors affecting the initial Qmax include the IPSS-storage symptom score, the prostate volume and a past history of AUR. Accordingly, in patients who have a higher IPSS-storage symptom score, a smaller prostate volume and a past history of AUR, there might be a detrimental effect on the improvement of the Qmax in the initial stage following the surgery. These factors might also be used as long-term prognostic factors for patients who undergo TURP or PVP.

\section{CONFLICT OF INTEREST}

No potential conflict of interest relevant to this article was reported.

\section{REFERENCES}

1. Wasson JH, Reda DJ, Bruskewitz RC, Elinson J, Keller AM, Hen- 
derson WG. A comparison of transurethral surgery with watchful waiting for moderate symptoms of benign prostatic hyperplasia. The Veterans Affairs Cooperative Study Group on Transurethral Resection of the Prostate. N Engl J Med 1995;332:75-9.

2. Park JS, Min GE, You CH, Hong B, Kim CS, Ahn H, et al. Comparison of treatment outcomes between photoselective vaporization and transurethral resection of the prostate for benign prostatic hyperplasia. Korean J Urol 2007;48:297-303.

3. Bachmann A, Schürch L, Ruszat R, Wyler SF, Seifert HH, Müller A, et al. Photoselective vaporization (PVP) versus transurethral resection of the prostate (TURP): a prospective bi-centre study of perioperative morbidity and early functional outcome. Eur Urol 2005; 48:965-71.

4. Barry MJ, Mulley AG Jr, Fowler FJ, Wennberg JW. Watchful waiting vs immediate transurethral resection for symptomatic prostatism. The importance of patients' preferences. JAMA 1988;259: 3010-7.

5. Jung PB, Lee JG. Prognostic value of pressure-flow study and clinical parameters in the outcome measurement after transurethral prostatectomy in patients with BPH. Korean J Urol 1999;40:1671-6.

6. Jung YS, Hwang TK, Kim JC. The outcome and satisfaction of patients with lower urinary tract symptoms/benign prostatic hyperplasia following transurethral resection of the prostate according to urodynamic obstruction and the bladder function. Korean J Urol 2007;48:965-70.

7. Kim HH, Kwak C, Seo SI, Chung H, Lee ES, Lee CW. The effects and complications of transurethral resection for benign prostatic hyperplasia: results of long-term follow-up. Korean J Urol 1996;37: 268-80.

8. Abrams PH, Farrar DJ, Turner-Warwick RT, Whiteside CG, Feneley RC. The results of prostatectomy: a symptomatic and urodynamic analysis of 152 patients. J Urol 1979;121:640-2.

9. Fitzpatrick JM, Mebust WK. Minimally invasive and endoscopic management of benign prostatic hyperplasia. In: Wein AJ, Kavoussi
LR, Novick AC, Partin AW, Peters CA, editors. Campbell-Walsh urology. 9th ed. Philadelphia: Saunders; 2007. p.2831-6.

10. Jensen KM, Bruskewitz RC, Iversen P, Madsen PO. Significance of prostatic weight in prostatism. Urol Int 1983;38:173-8.

11. Cockett AT, Barry MJ, Holtgrewe HL, Sihelnick S, Williams R, McConnell J. Indications for treatment of benign prostatic hyperplasia. The American Urological Association Study. Cancer 1992;70(1 Suppl):280-3.

12. Hakenberg OW, Pinnock CB, Marshall VR. Preoperative urodynamic and symptom evaluation of patients undergoing transurethral prostatectomy: analysis of variables relevant for outcome. BJU Int 2003;91:375-9.

13. Im JG, Kim JC, Hwang TK. The urodynamic findings of male patients with LUTS and the effects of the pre-operative parameters on the outcome of transurethral resection of the prostate. Korean J Urol 2005;46:1256-61.

14. Chang HS, Park CH, Kim CI. Transitional zone volume: a predictor of acute urinary retention in patients with benign prostatic hyperplasia. Korean J Urol 2005;46:259-63.

15. Jang CS, Cho JS, Chung BS. Post prostatectomy prognostic factors in patients with acute urinary retention due to benign prostatic hyperplasia. Korean J Urol 2004;45:998-1001.

16. Cho JS, Kim CI, Seong DH, Kim HS, Kim YS, Kim SJ, et al. Relationship between serum prostate-specific antigen and prostate volume in men with benign prostatic hyperplasia from multicenter study. Korean J Urol 2005;46:792-8.

17. Kim SG, Han WC, Jeong HJ, Rim JS. The Influence of prostatic calcification and lymphocyte infiltration on the result of TURP in patients with benign prostatic hyperplasia. Korean J Urol 2003;44: 82-6.

18. Lee C, Jeon Y, Lee N. The correlation between Transition Zone Index, versus IPSS and Peak Flow Rate after transurethral resection of prostate in benign prostatic hyperplasia. Korean J Urol 1999;40: $1318-22$. 